\title{
FFP14 Symposium Synopsis
}

\section{Eric Kajfasz}

Centre de Physique des Particules de Marseille

Aix Marseille Université, CNRS/IN2P3, CPPM UMR 7346

163, avenue de Luminy, Case 902

13288 Marseille cedex 09, France

E-mail: kajfasz@cppm.in2p3.fr

\section{Thierry Masson}

Centre de Physique Théorique

Aix Marseille Université, Université de Toulon, CNRS, UMR 7332,

163 Avenue de Luminy, Case 907

13288 Marseille cedex 09, France

E-mail: thierry.massonecpt.univ-mrs.fr

\section{Roland Triay}

Centre de Physique Théorique

Aix Marseille Université, Université de Toulon, CNRS, UMR 7332,

163 Avenue de Luminy, Case 907

13288 Marseille cedex 09, France

E-mail: roland.triaydcpt.univ-mrs.fr

Frontiers of Fundamental Physics 14 - FFP14,

15-18 July 2014

Aix Marseille University (AMU) Saint-Charles Campus, Marseille 


\section{Foreword}

The $14^{\text {th }}$ annual international symposium "Frontiers of Fundamental Physics" (FFP14) was organized by the Labex OCEVU; it was held at the Faculty of Sciences of Aix Marseille University (AMU) July 15th-18th 2014. In France, Marseille is the second largest city and the oldest; Greek sailors from Phocaea under the name of Massalia founded it around $600 \mathrm{BC}$.

The FFP14 is the fourteenth in the series of FFP Symposium that began in India in 1997 with Prof. B.G. Sidharth and it became itinerant in 2004, through Europe, Canada and Australia. It covers topics in fundamental physics with the objective to enable scholars working in related areas to meet on a single platform and exchange ideas. In addition to highlighting the progress in these areas, the symposium invites the top researchers to reflect on the educational aspects of our discipline. Moreover, the scientific concepts are also discussed through philosophical and epistemological viewpoints. Several eminent scientists, such as the laureates of prestigious awards (Nobel, Fields Medal,...), have already participated in these meetings.

The FFP14 has been developed around seven main themes: Astroparticle Physics, Cosmology, High Energy Physics, Quantum Gravity, Mathematical Physics, Physics Education, Epistemology and Philosophy. The mornings were devoted to the plenary session, with talks for large audience of physicists in its first half and more specialized in its second half. The parallel session of the Symposium took place during the afternoon with seven thematic conferences and an additional one on open topics named "Frontiers of Fundamental Physics". These conferences were organized with the contributions of participants, in addition of the ones of invited speakers. Almost 300 scientists attended this multidisciplinary symposium in which young scientists were encouraged to present posters.

\section{The Committees}

\subsection{Local Organizing Committee}

\section{Board}

- Cyrille Baudouin (CPPM)

- Eric Kajfasz (CPPM)

- Catherine Levet (CPT)

- Thierry Martin (CPT)

- Thierry Masson (CPT)

- Jocelyne Munoz (CPPM)

- Angélique Pepe (CPPM)

- Roland Triay - Chair (CPT)

\section{Astroparticle Physics}

- Emmanuel Nezri (LAM)

- George Vasileiadis (LUPM)

\section{Cosmology}

- Brahim Lamine (IRAP) 
- Christian Marinoni (CPT)

\section{High Energy Physics}

- Lorenzo Feligioni (CPPM)

- Jean Loïc Kneur (L2C)

\section{Quantum Gravity}

- Simone Speziale (CPT)

- Francesca Vidotto (Radboud University Nijmegen)

\section{Mathematical Physics}

- Thomas Krajewski (CPT)

- Thierry Masson (CPT)

\section{Physics Education}

- Jacques Ginestié (ESPE)

- José Busto (CPPM)

\section{Epistemology and Philosophy}

- Marc Lachièze-Rey (APC)

- Julien Bernard (University of Konstanz \& CEPERC-AMU)

\section{Frontiers of Fundamental Physics}

- Roland Triay (CPT)

- Burra Sidharth (BMBSC)

\subsection{International Advisory Committee}

- Felix Aharonian (MPIK Heidelberg)

- Aurelien Barrau (Univ. Joseph Fourier Grenoble)

- Claudia De Rham (Case Western Reserve University)

- Luigi Del Debbio (University of Edinburgh,)

- Zoltan Fodor (Bergische Universität Wuppertal)

- Klaus Fredenhagen (Universität Hamburg)

- Tim Gershon (University of Warwick, Coventry)

- Christophe Grojean (CERN)

- Luigi Guzzo (Osservatorio di Brera)

- Eric Kajfasz (CPPM)

- Julien Lavalle (LUPM, OCEVU)

- Giovanni Landi (Univ. Trieste)

- Martin Lemoine (IAP)

- Jerzy Lewandowski (University of Warsaw)

- Marisa Michelini (University of Udine) 
- Aleandro Nisati (CERN)

- Adi Nusser (Israel Institute of Technology)

- Aurelio Juste Rozas (IFAE, Universitat Autonoma de Barcelona)

- Roland Triay - Chair (CPT)

- Jean-Philippe Uzan (IAP)

- Filippo Vernizzi (IPhT/CEA Saclay)

- Martin White (Univ. California, Berkeley)

\subsection{International Scientific Committee}

- Abhay Ashtekar (Penn State Univ.)

- François Bouchet (IAP)

- Alain Connes (Collège de France)

- Nathalie Deruelle (APC)

- Francois Le Diberder (Univ. Pierre et Marie Curie)

- Christopher T. Sachrajda (Univ. of Southampton)

- Pierre Salati (Univ. de Savoie)

- Bernard Sadoulet (IMPAC, UC Berkeley)

- Joe Silk (Univ. Oxford)

- Alexei Starobinsky (Landau Institute)

- Andrew W. Strong (MPE, Garching)

\subsection{International FFP Committee}

- Claude Cohen-Tannoudji (Collège de France) - Hon. Joint Chairman

- David Finkelstein (Georgia Tech)

- Walter Greiner, (IAS, Frankfurt)

- Marc Lachièze-Rey (APC)

- Douglas D. Osheroff (Stanford Univ.) - Hon. Joint Chairman

- Fernando Quevedo (ICTP, Trieste)

- B.G. Sidharth (B.M.Birla Science Centre) - Convenor

\section{Scientific content}

These last years have culminated in major results in fundamental physics, and in particular the discovery of the Higgs boson and the measurement of the fluctuations of the blackbody spectrum temperature of the Cosmic Microwave Background. The first one reinforces the standard model of elementary particle physics and the second one provides a basis to the inflation scenario and the quantum origin of cosmological fluctuations, the seeds required for the formation of cosmological structures, and favours a non-zero cosmological constant or/and the alternatives named Dark Energy.

While describing phenomena at extremely large scale for the one and extremely small scale for the other one, the advances in these fields of physics spawn us a common path to go deeper and deeper in the understanding of fundamental laws. The next steps to accomplish are the correct extension of the Standard Model of particle physics above the electroweak scale, the identification 
of the Dark Matter and of the Dark Energy, the quantum description of gravity, as well as the development of new mathematical structures related to these questions.

These issues were jointly discussed in the plenary parts of the Symposium, while recent developments and ongoing researches were debated on dedicated conferences, whose aims are more precisely described hereafter. The multidisciplinary aspect of the symposium will be kept in an additional conference "Frontiers of Fundamental Physics", whose main topics were identified all along the submission procedure. It addresses issues of fundamental physics that are not included in the other seven conferences of the symposium.

\subsection{Astroparticle Physics (High energy phenomena in the Universe)}

At the boundaries of particle physics, cosmology and astrophysics, the astroparticle physics field is a very active domain of fundamental physics both on experimental and theoretical sides. The field focuses on elementary particles in astrophysical or cosmological contexts and adresses questions like the understanding of the cosmic rays, the dark matter enigma, the matter-antimatter asymmetry of the universe.

If the standard LambdaCDM cosmological model indicate the presence of a large amount of non-baryonic dark matter in the universe, the true nature, the behaviour and the precise distribution of this unknown specie remain illusive and is one of the major question of modern physics. These dark matter is tracked by multi-messenger astronomy and underground experiments.

Furthermore, the cosmic rays are also a long standing but fundamental question and understanding their origin, their sources and their propagation are key issues to fully control the features of the CR spectra.

Modelling and measuring cosmic ray fluxes of different messengers like gammas neutrinos, positrons, antiprotons are determining issues for dark matter identification, as well as for the understanding of violent phenomena like gamma ray bursts and supernovae remnant emissions and their contribution to the cosmic ray spectrum. Moreover those phenomena provide probes of the galactic and extragalactic universe.

The present era brings an precedent level of modelling and precision measurements with experiments (like FERMI, HESS, PAMELA, AMS, ICECUBE, ANTARES, XENON, LUX...) to progress in these interdisciplinary fields.

The purpose of the conference was to bring together scientists of adjoining fields to exchange or confront ideas and results.

The main topics are: Dark Matter, Cosmic Rays, Astrophysical jets, Multimessenger and Multiwavelength astronomy, Galactic and Extragalactic emissions, and Neutrinos.

\subsection{Cosmology (The accelerating universe: spacetime structure and matter structures)}

Dark energy cosmology, the study of the causes and effects of the accelerated expansion of the universe, is the natural crossroad where fundamental physics, astrophysics and particle physics meet. This interdisciplinary character has enormously accelerated the progress of researches in this field, and exciting challenges and breakthroughs are expected in the next decade.

Reveling the finest details of cosmic acceleration, unveiling the effects of dark energy on structure formation and evolution processes, and advancing dark energy studies to the next level of 
complexity, critically depend on the ability to increase the sensitivity of our instruments, to design efficient observational probes and to remove the potential astrophysical biases that can limit our ultimate understanding of the phenomenon. In the next decade, several major ground- and spacebased experiments are expected to take up the challenge by drawing a realistic portrait of this elusive component and by answering fundamental questions such as: what is the physical nature of dark energy? Is it a static component or a dynamical one of the cosmic landscape? Is General Relativity the correct theory of gravity on the scales of the Universe? Is the standard cosmological paradigm consistent or are there glimpses of new physics?

The purpose of the conference was to bring together observers, modelers and theorists in order to understand the strengths, weaknesses, and complementarities of current and planned dark energy probes as well as of the theoretical approaches developed to interpret and predict observations.

The aim was to present and discuss the latest research results in the fields, as well as to indicate new paths for future explorations by sharing expertise on the following topics: how to design future observational probes of dark energy and optimize the synergy between them? What are the best observables to characterize dark energy? How best to constrain the values of the fundamental cosmological parameters? What are the most efficient and universal ways to parameterize eventual deviations from the Friedman model and to discriminate among competing theories of modified gravity

The main topics are: Dark Energy - Dark Gravity, Inflation, Primordial Non-Gaussianity, Cosmological parameters, Cosmic Microwave Background, Large Scale Structure of the Universe, Galaxy formation and evolution, Galaxy clustering, Gravitational Lensing, Redshift surveys

\subsection{High Energy Physics (Beyond the Standard Model, Progress in Lattice QCD, Heavy Ion physics)}

The standard model (SM) of the strong, weak, and electromagnetic interactions gives a complete description of the elementary particles and their interactions, accurately describing present data up to the highest presently available TeV scale energies at the LHC. This success culminated with the 2012 discovery of a Higgs-like boson by the LHC ATLAS and CMS collaborations. However, the SM has serious limitations, and the particle physics community is now confronted with the need to identify the correct extension of the SM above the electroweak scale, allowing for a more natural electroweak symmetry breaking (EWSB).

Indeed, the scalar sector effective potential could be the manifestation of a more fundamental mechanism with a dynamical explanation for the EWSB. The two main scenarios for a more natural EWSB mechanism, addressing the hierarchy and naturalness problems, are either low energy supersymmetry (SUSY) or a TeV scale strongly interacting sector. Also of crucial importance to any BSM scenario are the quark and lepton flavor issues, namely the consistency and possible dynamical explanation of the observed pattern of quark masses and mixing as well as neutrino masses and mixing. The direct searches of SUSY signals at the LHC will continue to be crucial to constrain the many SUSY scenarios presently considered. Alternatively, the precise measurements of all the available Higgs decay modes at the LHC will be crucial to disentangle the many strongly interacting scenarios.

On the other side, the dark matter (DM) problem is still unsolved. There are strong theoretical arguments in favor of a "particle physics" solution within the previous context beyond the SM. A 
generic DM particle candidate in these theories is the so-called WIMP (weakly interacting massive particle). Complementarily to direct and indirect detection experiments, the LHC also gives hope that if a DM particle is discovered with parameters accessible to it, it will be possible to place this particle into a coherent particle physics scenario.

The symposium will also aim to discuss recent progress in lattice QCD and, more generally, lattice field theory computations. These play a critical role in our understanding of important nonlinear phenomena, ranging from phase transitions in the early universe and in very dense stars to the binding of quarks and gluons into hadrons and nuclei, or from a possible substructure for the Higgs boson to very small effects that may reveal new fundamental physics in experiments.

Another topics aimed to be addressed is Heavy Ion physics, after the first LHC results from the ALICE collaboration and recent theoretical developments on the status of the quark gluon plasma and the deconfinement transition.

The main topics are: Beyond the Standard Model (scenarios, LHC constraints and prospects), Supersymmetric Models, Dynamics of Electroweak Symmetry Breaking, Quark and Lepton Flavor physics and issues, Neutrino Physics and Models, Progress in Lattice QCD (zero and finite temperature), Heavy Ion physics, Dark matter (candidates and models)

\subsection{Quantum Gravity (Mathematical methods and physical applications)}

Quantum gravity remains one of the major open problems at the foundation of physics. There is not yet a consensus about its solution, but the last years have seen substantial progress both in the direction of the possibility of measurements at the Planck scale and on the theoretical side.

For example, the MAGIC observations of the possible non-trivial dispersion relations for light and the astrophysical analysis of the Crab nebula, for instance, have shown that Planck scale observations are possible, contrary to what generally assumed until a few years ago, and have essentially ruled out some tentative quantum gravity theories predicting certain Lorentz violations at the Planck scale.

On the theoretical side, the proof of the finiteness of the loop gravity amplitudes at all orders and the evidence about their small- $\hbar$ limit put the theory on a ground much solid than a few years ago. The calculations of possible quantum gravity signatures on the CMB connects these the theoretical advance to cosmology.

The main topics are: Loop Quantum Gravity, Background-Independent Approaches, Black Hole Entropy, Loop Quantum Cosmology, Twistor theory

\subsection{Mathematical Physics (Seeking of foundations)}

In the past, fundamental problems in theoretical physics have stimulated profound breakthroughs in mathematics, and the current description of the fundamental interactions of particle physics, as well as the description of the whole Universe, rely heavily on geometric and algebraic mathematical structures: representation theory, gauge theories, Riemannian geometry, operator algebras, invariants, combinatorial algebras...

Some recent research programs in mathematical physics have the ambition to answer some unsolved questions of today's description of Nature, for instance the search for a theory of the quantum gravity, or the mathematical structure of the standard model (SM) of particle physics... 
The new mathematical structures that have emerged so far take their roots in both geometry and algebra, and they make apparent some new structures connecting the two. For instance, new mathematical frameworks for gauge field theories, or new quantization procedures have been recently proposed.

As an illustration, the last thirty years have seen the spectacular development of noncommutative geometry (NCG), whose objective is to propose a mathematical framework in which it is possible to think of geometry in terms of operator algebras. On the mathematical side, NCG has provided some new profound results and has stimulated the construction of new structures, while on the physical side, it proposes to take a fresh look at the mathematics behind the SM.

Moreover, the last decade has witnessed important developments in our understanding of the mathematical structure behind renormalisation in quantum field theory. In particular, these include the construction of combinatorial Hopf algebras underpinning the renormalisation procedure and the realisation of gauge symmetries.

The main topics are: Quantization (geometric and algebraic methods), Noncommutative geometry (foundation and applications), and Renormalization (algebraic and analytic aspects).

\subsection{Physics Education (Teaching and learning science: from general education to high level university education)}

Nowadays, scientific research is at the heart of many stakes of local developments, as well as territorial and international ones, and it is found in several strategies. Paradoxically, students desert many scientific domains; they prefer some professionalizing training that could provide them more opportunities in terms of employment after graduation. This disaffection seems going together with a decrease of motivation, even a decrease of student's academic level in scientific disciplines. In master level courses, it is therefore not unusual to be confronted with important differences between, on the one hand, students' expectations, sometimes manifested by a lack of interest, low motivation and work which sometimes appears as minimalist as possible, and, secondly, the requirements of these courses in knowledge, commitment, or in simply in scientific culture.

Among the issues that student behavior generated in the scientific community, particularly two of them will be discussed in this conference:

1. The organization of studies in the LMD structure modifies significantly the training curriculum of students: reduction of volumes of teachings, sequential organization less recurrent, open choice of very diverse options, etc. Is that correct to think that this evolution creates a gap between the structural organization of university curricula and the methods of knowledge transfer that are perhaps no longer suitable to these new structures?

2. The stakes and motivations of students have strongly evolved in recent years, the social goals of university studies have become as important as the domains studied. Their professional future is a criterion more and more important in choosing training and courses. However, the structure of university training, which goes from the general to the specialized specific area, does not give all the desired goals readability. Is this lack of concordance the cause of the significant drop in motivation for students who do not see very well "what's the point."

The expected contributions should provide us with some insights, elements of understanding on the impact of changing organizational structures of university education. They should also out- 
line issues to explore new educational organizations to think and build in order to improve the effectiveness of the teaching-learning in higher education in the fields of physical science education.

The main topics are: Teaching sciences at University and new pedagogies, teaching sciences at the secondary school, science education at school, promotion of the scientific culture through sciences associations.

\subsection{Epistemology and Philosophy}

The goal is to discuss the epistemological and ontological status of the various notions, concepts involved in modern physics.

This concerns first the physical theories and models themselves: what is a physical theory? What is a good physical theory? In particular how can we characterize it beyond its possibly multiple mathematical formulations? How to interpret the fact that an unique theory can be described by very different mathematical tools and, more generally, how to answer to the Wigner question about the "unreasonable efficiency of mathematics for physics"?

Also, the relations between distinct theories cannot be reduced to differences of mathematical formulations (since an unique theory may admit different formulations). This suggests two tracks:

- try to analyze the theories, their differences, and also their evolutions independently of any mathematical formulations. Clearly a task for epistemology and philosophy!

- search for a mathematical formalism sufficiently general to encompass very different theories (presently formulated with different mathematical tools).

Symplectic geometry, groups and algebras with their generalizations play an important role in that concern. We want to discuss in what extent the theory of category may also be useful. We will show particular interest toward the analysis of the evolution of theories (e.g., from classical to quantum; or from Newtonian to Einsteinian physics), in the light of group or algebra deformations, or of categorization.

The main topics are: space, time and space-time, interpretation of Quantum Physics, observation process, and Realism.

\section{Social Events}

The welcome party took place at Officers' Mess restaurant at Fort Ganteaume, a privileged site that overlooks the old harbor. The cocktail was followed by 30 minutes of a spectacular firework that was organized by the Marseille City Hall for the French national fest. This was an unforgettable moment, a common feeling shared among all the participants.

For bringing an historical dimension to this event, the banquet was organized in one of prestigious dining rooms at the Palais du Pharo. It is a site whose construction was ordered by Napoleon III for Empress Eugenie in the second half of the nineteenth century; the references to classical architecture are numerous. From the gardens, one enjoys a nice overlooking to the Fort Saint-Jean and the harbor of Marseille.

The city of Marseille has welcomed the participants with a cocktail at the City Hall Thursday. After an official speech, participants were able to discover and enjoy some local specialties. Such 
an opportunity has been repeated for the duration of the symposium in the restaurants of the city, especially around the old harbor. Mediterranean crossroads of civilizations, Marseille's population is cosmopolitan and such a characteristics enriches the local gastronomy.

A public lecture in French (Les bâtisseurs du ciel : de Copernic à demain, Jean-Pierre Luminet) has been held at the Campus Saint Charles of the Aix-Marseille University to the attention of the local population, which has been immensely appreciated.

\section{Participants}

Tim AdAmo - Dep. of Applied Mathematics \& Theoretical Physics, Univ. of Cambridge, Cambridge, United Kingdom Alexander AFRIAT - Université de Bretagne Occidentale, Brest, France

Imen AL SAMARAI - Institut de physique nucléaire d'Orsay, Orsay, France

Lasma ALBERTE - Ben-Gurion University, Beer Sheva, Israel

Emanuele ALESCI - Warsaw University, Warszawa, Poland

Sergey Alexandrov - Laboratoire Charles Coulomb, Université Montpellier 2, Montpellier, France

Mikhail ALtAISKY - Space Research Institute RAS, Moscow, Russian Federation

Elena AMATO - INAF-Osservatorio Astrofisico di Arcetri, Firenze, Italy

Seramika ARI WAHYOEdi - Centre de Physique Theorique de Marseille, Marseille, France

Paolo AsChIERI - Dipart. di Scienze e Innovazione Tecnologica, Univ. del Piemonte Orientale, Alessandria, Italy

Mehdi Ass ANIOUSSI - Institute of Theoretical physics, University of Warsaw, Warsaw, Poland

Pierre ASTIER - LPNHE/CNRS/UPMC, Paris, France

Eric Audureau - Centre d'Epistémologie et d'Ergologie Comparative, Aix-en-Provence, France

Benjamin BAHR - Institute for Theoretical Physics, University of Hamburg, Hamburg, Germany

Cécile BarbachouX - ESPE, Université de Nice Sophia-Antipolis, Nice, France

Cyrille BAUdouin - Centre de physique des particules de Marseille, Marseille, France

Eric BAUSS AN - Institut Pluridisciplinaire Hubert Curien, Strasbourg, France

Julien BEL - Osservatorio Astronomico di Brera, Merate, Italy

Jibril Ben ACHOUR - Astroparticles \& Cosmology, Paris, France

Veniamin BEREZINSKY - INFN, Gran Sasso Science Institute, L'Aquila, Italy

Julien BERNARD - University of Konstanz-Zukunftskolleg and CEPERC, Konstanz, Germany

Paolo BertozZINI - Department of Mathematics and Statistics, Thammasat University, Khlongluang, Thailand

Fabien Besnard - Ecole Polytechnique Féminine, Sceaux, France

Eugenio BIANCHI - The Pennsylvania State University, University Park, United States

Pierre BIELIAVSKY - Université Catholique de louvain, Louvain la Neuve, Belgium

Alessandro BISIO - Università degli studi di Pavia, Dipartimento di Fisica, Pavia, Italy

Jean-Paul BLAIZOT - Institut de Physique Théorique Saclay, Gif-sur-Yvette, France

Alain BLANCHARD - Institut de recherche en astrophysique et planétologie, Toulouse, France

Alain BLONDEL - Dép. de Physique Nucléaire et Corpusculaire, University of Geneva, Genève, Switzerland

François BOUCHET - Institut d'Astrophysique de Paris, CNRS \& Sorbonne Universités-UPMC, Paris, France

Frédéric BOUQUET - Université Paris Sud, Orsay, France

Philippe BRAX - Institut de Physique Théorique, Gif sur Yvette, France

Christian Brouder - Institut de Minéralogie, de Physique des Matériaux et de Cosmochimie, Paris, France

Jean Philippe BRUnETON - Laboratoire Univers et Théories, Meudon, France

Juergen Brunner - Centre de Physique des Particules de Marseille, Marseille, France

John Bulava - Trinity College, Dublin, Dublin, Ireland

Igor BULYZHENKOV - Moscow Institute of Physics and Technology, Dolgoprudny, Russian Federation

Alexander BURInSKII - Theor. Phys. Lab., Nuclear Safety Inst., Russ. Acad. of Sciences, Moscow, Russian Federation

Damir BusKULIC - Université de Savoie, LAPP, Annecy-le-Vieux, France 
Jose Busto - Centre de Physique des Particules de Marseille, Université d'Aix-Marseille, Marseille, France Andrei B YKOV - Ioffe Institute of Physics and Technology, St. Petersburg, Russian Federation Anatael CABRERA - CNRS/IN2P3 - APC Laboratory, Paris, France Enrico CALLONI - Università Federico II - Napoli, Naples, Italy Philippe CAPONIS - Lebanese American University, Beirut, Lebanon Chiara CAPRINI - Institut de Physique Théorique, Gif sur Yvette, France John CARR - Centre de Physique des Particules de Marseille, Marseille, France Sylvain CARrozza - Centre de Physique Théorique, Marseille, France Gabriel CATREn - Laboratoire Sciences, Philosophie, Histoire, Univ. Paris Diderot, Paris, France Ángel José Chacón Velasco - Universidad Pedagógica y Tecnológica de Colombia, Bucaramanga, Colombia Ali Chamseddine - American Univ. of Beirut \& Inst. des Hautes Etudes Scientifiques, Bures-sur-Yvette, France Jérôme CHARLES - Centre de Physique Théorique, Marseille, France

Dyaa CHBIB - Centre de Physique Théorique, Marseille, France

Goffredo CHIRCO - Centre de Physique Théorique - Université Aix-Marseille, Marseille, France Inyong CHO - Seoul National University of Science and Technology, Seoul, Korea, Republic of Ricardo Coelho - Faculdade de Ciencias da Universidade de Lisboa, Lisbon, Portugal

Benjamin COUTURIER - Organisation Européenne pour la Recherche Nucléaire, Geneva, Switzerland Paschal COYLE - Centre de physique des particules de marseille, Marseille, France

Gabriella CROCCO - CEPERC UMR 7304, Aix-Marseille Université, Marseille, France

Sabine CRÉPÉ-RENAUdin - Laboratoire de Physique Subatomique et de Cosmologie de Grenoble, Grenoble, France Sam CUNLIFFE - Imperial College London, London, United Kingdom

Francesco D'ANDREA - University of Naples Federico II, Naples, Italy

Frédéric DAIGNE - Institut d'Astrophysique de Paris, Paris, France

Jeremy DALSENO - HH Wills Physics Laboratory, University of Bristol, Bristol, United Kingdom

Andrea DAPOR - Faculty of Physics, University of Warsaw, Warsaw, Poland

Amilcar DE QueIROZ - Universidade de Brasília \& Universidad de Zaragoza, Zaragoza, Spain

Cédric DefFAYET - Institut d'Astrophysique de Paris and Institut des Hautes Études Scientifiques, Paris, France

Alice DELSERIEYs - Aix-Marseille University - ESPE, Marseille, France

Pasquale Di BARI - University of Southampton, Southampton, United Kingdom

Cristinel Diaconu - Centre de Physique des Particules de Marseille, Marseille, France

Antonaldo DiAferio - Università di Torino, Dipartimento di Fisica, Torino, Italy

Sara Diglio - Centre de Physique de Particule de Marseille, Aix-Marseille Univerity, Marseille, France

Farès DJAMA - Centre de Physique des Particules de Marseille, Marseille Cedex 09, France

Arache Duannati-Atai - Arache Djannati-Atai, Paris, France

Samia DRAPPEAU - Institut de Recherche en Astrophysique et Planétologie, Toulouse, France

Eve-Aline DuboIS - University of Namur, Néchin, Belgium

Christian Duval - Centre de Physique théorique, Marseille, France

Valeriy Dvoeglazov - Universidad Autónoma de Zacatecas, Zacatecas, Mexico

Vladimir DZHUnUshaLIEV - Kazakh National University, Almaty, Kazakhstan

Bogar Díaz JimÉneZ - Benemérita Universidad Autónoma de Puebla, Puebla, Mexico

Michal ECKSTEIN - Jagellonian University, Krakow, Poland

Chang-Young EE - Sejong University, Seoul, Korea, Republic of

Semiha EFE - Anadolu Üniversitesi, Fen Bilimleri Enstitüsü, Eskişehir, Turkey

Kevin FALK - Centre de Physique Théorique, Marseille, France

Benoit FAMAEY - Observatoire astronomique de Strasbourg, Strasbourg, France

Martin FEIX - Israel Institute of Technology, Haifa, Israel

Lorenzo Feligioni - Centre de Physique de Particule de Marseille, Marseille, France

Agnès FERTÉ - Institut d'Astrophysique Spatiale, Orsay, France 
Plamen FIZIEV - Jount Institute of Nuclear Research, Dubna, Russian Federation Jordan FrAnÇOIS - Centre de Physique Théorique, Marseille, France

Màrius Josep Fullana I ALfOnso - Inst. de Mat. Multidisciplinària - Univ. Politècnica de València, València, Spain Cohl FUREY - Perimeter Institute for Theoretical Physics, Waterloo, Canada

Remo GARATTINI - University of Bergamo, Dalmine, Italy

Miguel Ángel García ArIZA - Benemérita Universidad Autónoma de Puebla, Puebla, Mexico

Esthere GARnIER - Centre de Physique des Particules de Marseille, Marseille, France

Jules GASCON - Institut de Physique Nucleaire de Lyon, Villeurbanne, France

Enrique GAZTAÑAGA - Instituto de Ciencias del Espacio, Bellaterra, Spain

Jacques GINESTIÉ - ESPE, Aix-Marseille Université, Marseille, France

Gabriel GiovannetTi - Centre d'Epistémologie et d'Ergologie Comparative, Paris, France

Damien GIVRY - ESPE, Aix Marseille Université, Marseille, France

Andrea GoldwURM - AstroParticule et Cosmologie, Paris, France

Diego GonZÁLEZ - Centro de Investigación y de Estudios Avanzados, Mexico City, Mexico

Bruno GonÇALVES - Inst. Federal de Educação, Ciência e Tecnologia do Sudeste de Minas Gerais, Juiz de Fora, Brazil Julien GrAIN - Institut d'Astrophysique Spatiale, Orsay, France

Walter GREINER - Frankfurt Institute for Advanced Studies, Frankfurt am Main, Germany

Luigi GuZzo - Istituto Nazionale di Astrofisica, Osservatorio Astronomico di Brera, Merate, Italy

Zbigniew HABA - Institute of Theoretical Physics, University of Wroclaw, Wroclaw, Poland

Hal Haggard - Centre de Physique Theorique, Marseille, France

Francis HALZEN - University of Wisconsin-Madison, Madison, United States

Jaume HARO - Universitat Potitècnica de Catalunya, Barcelona, Spain

Alan HeAVENS - Imperial College London, London, United Kingdom

Paula HERON - University of Washington, Seattle, United States

Céline Hess - Centre de Physique Théorique, Marseille, France

Philipp Hoenn - Perimeter Institute for Theoretical Physics, Waterloo, Canada

Christian Hoelbling - Bergische Universität Wuppertal, Wuppertal, Germany

Cyril Hugonie - Laboratoire Univers et Particules de Montpellier, Montpellier, France

Lam HUI - Columbia University, New York, United States

Jai-chan HwANG - Kyungpook National University, Daegu, Korea, Republic of

Patrick IgLesias-Zemmour - Institut de Mathématique de Marseille, CNRS, Marseille, France

Stéphane ILIC - Institut de Recherche en Astrophysique et Planétologie, Toulouse, France

Bruno Iochum - Centre de Physique Théorique, Marseille, France

Kiran Joshi - The University of Manchester, Manchester, United Kingdom

Eric KaJfasz - Centre de Physique des Particules de Marseille, Marseille, France

Igor KANATCHIKOV - National Quantum Information Center in Gdansk, Sopot, Poland

Nikolas KAUER - Royal Holloway, University of London, Egham, United Kingdom

Alexander KegEles - Max-Planck-Institute for gravitational physics (Albert-Einstein-Institut), Potsdam, Germany

Andrei KhMELnitsky - Ben-Gurion University of the Negev, Beer-Sheva, Israel

Jean-Loic KNEUR - Laboratoire Charles Coulomb, Montpellier, France

Joseph KouneIHER - Université de Nice Sophia Antipolis, Nice, France

Kazuya KoYAMA - University of Portsmouth, Portsmouth, United Kingdom

Thomas KRAJewsKI - Centre de Physique Theorique, Marseille, France

Piret KUUSK - Institute of Physics, University of Tartu, Tartu, Estonia

Kyujin KWAK - Ulsan National Institute of Science and Technology, Ulsan, Korea, Republic of

Marc LACHIÈZE-REY - Astroparticule et Cosmologie, Paris, France

Thomas LACROIX - Institut d'Astrophysique de Paris, Paris, France

Brahim LAMINE - Institut de recherche en astrophysique et planétologie, Toulouse, France 
Julien Lavalle - Laboratoire Univers et Particules de Montpellier, Montpellier, France Serge LAZZARINI - Centre de Physique Théorique, Marseille, France

Olivier LE FÈVRE - Laboratoire d'Astrophysique de Marseille, Marseille, France

Gandalf LECHNER - Institute for Theoretical Physics, University of Leipzig, Leipzig, Germany

Laurent LELlOUCH - Centre de Physique Theorique, Marseille, France

Catherine Levet - Centre de Physique Théorique, Marseille, France

Jerzy LEWANDOwSKI - Uniwersytet Warszawski, Warszawa, Poland

Linda LinSEFORS - Laboratoire de Physique Subatomique et de Cosmologie, Grenoble, France

Daniel Litim - Department of Physics and Astronomy, University of Sussex, Brighton, United Kingdom

Etera LIVINE - ENS Lyon - Laboratoire de Physique - CNRS UMR 5672, Lyon, France

Fedele LIZZI - Università di napoli Federico II, Napoli, Italy

Vladimir LuKOVIC - University of Rome Tor Vergata, Rome, Italy

Jean-Pierre Luminet - Observatoire de Paris, Meudon, France

Martín LóPEZ-CorRedoIRA - Instituto de Astrofísica de Canarias, La Laguna (Tenerife), Spain

Wolfgang MADER - Institut fuer Kern- und Teilchenphysik, Dresden, Germany

Stefano MAGNI - Laboratoir Univers et Particules de Montpellier, Montpellier, France

Fabio MaLtoni - Centre for Cosmology, Particle Physics and Phenomenology, Louvain-la-Neuve, Belgium

Pierre MANDRIN - , Wettingen, Switzerland

Antonino Marciano - Department of Physics, Fudan University, Shanghai, China

Alexandre MARCOWITH - Laboratoire Univers et Particules de Montpellier, Montpellier, France

Christian Marinoni - Centre de Physique Théorique, Marseille, France

Giovanni MAROzZI - Department of Theoretical Physics, University of Geneva, Geneva, Switzerland

Pierre MartinetTi - Università degli Studi di Napoli Federico II, Napoli, Italy

Jerome MARTIN - Institut d'Astrophysique de Paris, Paris, France

Thierry MARTin - Centre de Physique Théorique, Marseille, France

Thierry MASSON - Centre de Physique Théorique, Marseille, France

Sabino Matarrese - Dipartimento di Fisica e Astronomia G. Galilei, Università di Padova, Padova, Italy

Aurore Mathieu - Centre de Physique des Particules de Marseille, Marseille, France

Sergey MAYBUROV - Lebedev Institute of Physics, Moscow, Russian Federation

Noureddine MeBARKI - Constantine1 University, Constantine, Algeria

Federico MESCIA - Universitat de Barcelona, Barcelona, Spain

Marisa MICHELINI - URDF - DCFA, University of Udine, Udine, Italy

Jean-Philippe MICHEL - Université de Liège, Liège, Belgium

Salvatore Mignemi - Dipartimento di Matematica, Università di Cagliari, Cagliari, Italy

Elie MiLgrom - Université Catholique de Louvain, Louvain-la-Neuve, Belgium

Felix Mirabel - Commissariat à l'Energie Atomique et Energies Alternatives Saclay, Gif sur Yvette, France

Satoshi Mishima - University of Rome "La Sapienza", Roma, Italy

Pol Mollitor - Laboratoire d'Astrophysique de Marseille, Marseille, France

Olivier MORIZOT - Université Aix Marseille, Marseille, France

Valérie MUNIER - Université Montpellier 2 - Faculté d'éducation, Montpellier, France

Jocelyne MunOz - Centre de Physique des Particules de Marseille, Marseille, France

Jeff Murugan - Department of Mathematics \& Applied Mathematics, Cape Town, South Africa

Ilkka MÄKINEN - Faculty of Physics of the University of Warsaw, Warsaw, Poland

Jordi NADAL - II. Physikalisches Institut, Göttingen, Germany

Yoshikazu NAGAI - Centre de Physique des Particules de Marseille, Marseille, France

Christopher NEU - University of Virginia, Charlottesville, United States

Emmanuel NEZRI - Laboratoire d'Astrophysique de Marseille, Aix-Marseille University, Marseille, France

Hyerim NoH - Korea Astronomy and Space Science Institute, Daejon, Korea, Republic of 
Adi NUSSER - Israel Institute of Technology, Haifa, Israel

Robert OECKL - Centro de Ciencias Matemáticas, Universidad Nacional Autónoma de México, Morelia, Mexico

Daniele ORITI - Max Planck Institute for Gravitational Physics (Albert Einstein Institute), Golm, Germany

Antonio PADILLA - University of Nottingham, Nottingham, United Kingdom

Antonio PALAZZO - Max Planck Institut fuer Physik, Munich, Germany

Agostino PATELla - CERN and Plymouth University, Geneva, Switzerland

Guy PELletier - Institut de Planétologie et d'Astrophysique de Grenoble, Grenoble, France

Roser PELlo - Institut de Recherche en Astrophysique et Planétologie, Toulouse, France

Alejandro PEREZ - Centre de Physique Théorique, Marseille, France

Federico PIAzZA - Centre de Cosmologie Physique de Paris, Paris, France

Nicola PInAmONTI - University of Genova, Genova, Italy

Stefan POKORSKI - University of Warsaw, Faculty of Physics, Warsaw, Poland

Vladimir PTUSKIN - Inst. for Physical Science and Technology, University of Maryland, College Park, United States

Arnau Pujol - Institut de Ciències de l'Espai (ICE-IEEC/CSIC), Bellaterra, Spain

Jorge PULLIN - Louisiana State University, Baton Rouge, United States

Angélique PÈPE - Centre de Physique des Particules de Marseille, Marseille, France

Jérémie Quevillon - Laboratoire de Physique Théorique d'Orsay, Orsay, France

Sabir RAMAZANOV - Universite Libre de Bruxelles, Brussels, Belgium

Kasia REJZNER - University of York, York, United Kingdom

Aldo RIELlo - Centre de Physique Théorique de Luminy, Marseille, France

Brigitte RocCA - Institut Astrophysique de paris, Paris, France

Andrei RoDIN - Institute of Philosophy, Russian Academy of Sciences, Moscow, Russian Federation

Jérôme Rosanvallon - Université Paris Diderot, Paris, France

Daniel RouAn - Observatoire de Paris, Meudon, France

Carlo RovelLI - Université Aix-Marseille, Marseille, France

Alexander RUdolPh - California State Polytechnic University, Pomona, Pomona, United States

Paola RUGgIERO - Paola Ruggiero, Galatina, Italy

Chang-Mo RYU - Pohang University of Science and Technology, Pohang, Korea, Republic of

Heidi RzEHAK - Albert-Ludwigs-Universitaet Freiburg, Physikalisches Institut, Freiburg, Germany

David SANCHEZ - Laboratoire d'Annecy-le-Vieux de Physique des Particules, Annecy-le-Vieux, France

Xavier SARAZIN - Laboratoire de l'Accélérateur Linéaire, Orsay, France

Subir SARKAR - University of Oxford \& NBI Copenhagen, Oxford, United Kingdom

Frank SAUERESSIG - Institute for Mathematics, Astrophysics and Particle Physics, Nijmegen, Netherlands

Carol SCARLETT - Florida Agricultural and Mechanical University, Tallahassee, United States

Sandrine SCHLÖGEL - University of Namur, Namur, Belgium

Leonardo SENATORE - Stanford University, Stanford, United States

Tatyana SHESTAKOVA - Southern Federal University, Rostov-on-Don, Russian Federation

Burra SIDHARTH - B.M.Birla Science Centre, Hyderabad, India

Jan SIKORSKI - Faculty of Physics, University of Warsaw, Warsaw, Poland

Joe SILK - Institut d'Astrophysique de Paris, Paris, France

Vera Georgievna SinIts YNA - P. N. Lebedev Physical Institute, RAS, Moscow, Russian Federation

Vera Yurievna Sinits YNA - P. N. Lebedev Physical Institute, RAS, Moscow, Russian Federation

Vishagan SIVANESAN - Institut d'Astrophysique de Paris, Paris, France

Alessandro SPALLICCI - Université d'Orléans, Observatoire des Sciences de l'Univers, Orléans, France

David SPERGEL - Department of Astrophysical Sciences, Princeton University, Princeton, United States

Simone SpezIale - Centre de Physique Theorique, Marseille, France

Paraskevas SPHICAS - European Center for Particle Physics and University of Athens, Geneva, Switzerland

John STACHEL - Center for Einstein Studies, Boston University, Boston, United States 
Alexei Starobinsky - Landau Institute for Theoretical Physics RAS, Moscow, Russian Federation Alberto STEFANEL - University of Udine, Udine, Italy Heinrich STEIGERWALD - Aix-Marseille University, Marseille, France Christoph STEPHAN - Universität Potsdam, Institut für Mathematik, Potsdam, Germany Diego STOCCO - Subatech - Laboratoire de Physique Subatomique et des Technologies Associées, Nantes, France Roman SVERDLOV - Indian Institute of Science Education and Research, Mohali, India Jędrzej SwIEŻEwSKI - Faculty of Physics, University of Warsaw, Warsaw, Poland Kalman SZABO - Forschungszentrum Julich, Julich, Germany Jean-Jacques SzCZECINIARZ - Paris 7 University, Paris, France Li TAO - Laboratoire d'Annecy-le-Vieux de physique des particules, Annecy-le-Vieux, France Angelo TARTAGLIA - Politecnico di Torino and INFN, Torino, Italy Emanuele TASSI - Centre de Physique Théorique, Marseille, France Thomas TAVERNIER - Astroparticule et Cosmologie,universite Paris 7, Paris, France Pierre TAXIL - Aix-Marseille Université - Centre de Physique Théorique UMR 7332, Marseille, France Guo Chuan THIANG - University of Oxford, Mathematical Institute, Oxford, United Kingdom Johannes THÜRIGEN - Max-Planck Institute for Gravitational Physics (Albert-Einstein Institute), Potsdam, Germany Reiko TORIUMI - Centre de Physique Théorique, Marseille, France

Efrain TORRES-Lomas - Universidad de Guanajuato, León, Mexico Alessandro TosinI - Dipartimento di Fisica dell'Università degli Studi di Pavia, Pavia, Italy Guillaume TRAP - Palais de la découverte, Paris, France

Roland Triay - Centre de Physique Théorique, Marseille, France

Yasunobu UCHIYAma - Rikkyo University, Toshima-ku, Japan

Piero Ullio - Scuola Internazionale Superiore di Studi Avanzati, Trieste, Italy

Yuko URAKAWA - Institute for Advanced Research, Nagoya University, Nagoya, Japan

Laurent VACAVAnt - Centre de Physique des Particules de Marseille, Marseille, France

Galliano VALENT - Laboratoire de Physique Theorique et des Hautes Energies, Paris, France

Jose W.F VALlE - Instituto de Física Corpuscular, Paterna, Spain

Walter VAN SUIJLEKOM - Radboud University Nijmegen - IMAPP (Mathematics), Nijmegen, Netherlands

George VASILEIADIS - Laboratoire Univers et Particules de Montpellier, Montpellier, France

Gabriele VenezIANo - Collège de France, Paris, France

Murli Manohar Verma - Department of Physics, University of Lucknow, Lucknow, India

Filippo VerNIZZI - Institut de Physique Théorique - CEA, Gif-sur-Yvette, France

Francesca VIDOTTO - Radboud University, Nijmegen, Netherlands

Laurence VIEnNot - Univ Paris Diderot, Sorbonne Paris Cité, Paris, France

Alexander VIKMAN - Ludwig-Maximilians-Universität München, Munich, Germany

Jean-Marc VIREY - Centre de Physique Théorique, Marseille, France

Cristina VOLPE - Astroparticule et Cosmologie, Paris, France

David WANDS - Institute of Cosmology and Gravitation, University of Portsmouth, Portsmouth, United Kingdom

Gordon WATTS - University of Washington, Seattle, United States

Amanda Weltman - University of Cape Town, Cape Town, South Africa

Susanne WESTHOFF - University of Pittsburgh, Pittsburgh, United States

Bernard Whiting - University of Florida, Gainesville, United States

Wolfgang Wieland - Institute for Gravitation and the Cosmos, Penn. State University, University Park, United States

John WILliaMSON - University of Glasgow, Glasgow, United Kingdom

Edward WILSON-EwING - Louisiana State University, Baton Rouge, United States

Nadine WITKOws KI - Université Pierre et Marie Curie, Paris, France

Michael WrIGHT - The Archive Trust for Mathematical Sciences and Philosophy, Fougeres, France

Raimar WULKENHAAR - Math. Institut, Westfaelische Wilhelms-Universitaet Muenster, Muenster, Germany 
Marco ZARO - LPTHE, Université Pierre et Marie Curie, Paris VI, Paris, France Vaclav ZatloukAl - Fac. of Nucl. Sciences and Phys. Engineering, Czech Technical Univ., Praha 1, Czech Republic Antonia ZIPFEL - Wydzial Fizyki Uniwersytetu Warszawskiego, Warszawa, Poland

Hervé ZWIRN - Unité support au Consortium de Valorisation Thématique SHS, Meudon, France

\section{Acknowledgements}

The funding agencies, which made this conference possible, are:

- Labex OCEVU

- The Aix Marseille University

- Ville de Marseille

- Conseil général des Bouches du Rhône

- CNRS

- Ministère de l'Enseignement Supérieur et de la Recherche

- ESPE

- Labex ARCHIMEDE

- GDR Renormalization

- GDRE GREFI-GENCO

- FRUMAM

These grants were very welcome and we gratefully acknowledge such a financial support.

FFP14 was made possible thanks to the International FFP Committee who maintains this symposium alive and the International Scientific committee for guarantying an high scientific level. The keen interest and enthusiasm were due mainly to the judicious choice of subjects and speakers made by the members of the International Program Committee. Finally, the session chairpersons ensured an orderly conference in which discussions and questions played a central and stimulating role.

To all of them, on behalf on the Local Organizing Committee, we express our deep gratitude,

Eric Kajfasz, Thierry Masson and Roland Triay 